\title{
Philosophiques
}

\section{Remarques sur l'épistémologie française et l'épistémologie américaine}

\section{Jean Theau}

Volume 3, numéro 2, octobre 1976

URI : https://id.erudit.org/iderudit/203053ar

DOI : https://doi.org/10.7202/203053ar

Aller au sommaire du numéro

Éditeur(s)

Société de philosophie du Québec

ISSN

0316-2923 (imprimé)

1492-1391 (numérique)

Découvrir la revue

Citer cet article

Theau, J. (1976). Remarques sur l'épistémologie française et l'épistémologie américaine. Philosophiques, 3(2), 183-207. https://doi.org/10.7202/203053ar d'utilisation que vous pouvez consulter en ligne.

https://apropos.erudit.org/fr/usagers/politique-dutilisation/ 


\title{
REMARQUES SUR L'EPISTÉMOLOGIE FRANÇAISE ET L'EPISTÉMOLOGIE AMERICAINE
}

\author{
par Jean Theau
}

Les langues sont tout imprégnées de philosophie et chacune d'elles entraîne l'esprit à philosopher d'une certaine manière. Ainsi la philosophie grecque n'est passée que difficilement en latin et personne n'oserait jurer qu'elle y soit jamais passée tout entière. De même, pour nous en tenir à quelques schématiques exemples, des rapports se sont noués entre l'anglais et l'empirisme, le français et le cartésianisme, l'allemand et un certain type de rationalisme. Et la crainte n'est pas vaine que l'adoption d'une langue internationale en philosophie, pourtant remplie d'évidents avantages, n'impliquât l'adoption a priori d'un certain style de pensée qui aurait pour contrepartie l'exclusion de beaucoup d'autres . . . Mais nous laisserons de côté ce genre de problèmes, aussi délicats pour la théorie qu'épineux dans la pratique, et pour introduire les remarques qui suivent nous nous bornerons à rappeler un autre fait. Le rapport des philosophies suit souvent le rapport des langues : ou, en d'autres termes, l'influence d'une philosophie tient en général pour beaucoup à l'influence de la langue qui la porte, et cette dernière procède dans une large mesure de raisons politico-historiques qui n'ont pas une relation directe avec la valeur respective des philosophies. Tout le monde convient que c'eût été une perte irréparable pour l'Occident si la philosophie grecque s'était trouvée abandonnée quand la langue latine eut supplanté la langue grecque. Or des pertes du même genre, sinon de la même ampleur, menacent de se produire aujourd'hui en Amérique où ce qui n'a pas été soit traduit en anglais soit assimilé par la tradition anglophone devient très vite, surtout dans certains domaines comme la philosophie des sciences, à peu près complètement ignoré. ${ }^{1}$

1. Il faut ajouter, pour être juste, que la tendance à l'autarcie intellectuelle compensée par une curiosité largement électique envers tout ce qui compte à l'étranger ; et l'Amérique d'aujourd'hui, comme jadis l'Angleterre s'intéresse au monde entier. 
L'épistémologie française - entendez comme on l'entend dans notre langue la philosophie des sciences écrite en français - a connu jusqu'au milieu de ce siècle un prestige mérité, car elle n'avait cessé d'avancer sur la route que ses grands initiateurs, qui ne sont autres que Descartes et Pascal, les Encyclopédistes et August Comte, lui avaient ouverte. Mais le fait que beaucoup d'œuvres importantes du XXe siècle (ce qui est le cas pour une bonne partie des travaux épistémologiques de Brunschvicg, Lalande, Meyerson et Bachelard par exemple), joint au fait que ces auteurs se sont toujours refusés à parler ordinairement le langage de la logique moderne - habit de rigueur pour tant de nos contemporains - contribue à accréditer l'opinion que cette tradition épistémologique est désormais tombée en désuétude. Nous voudrions montrer ici ou plutôt suggérer par une ou deux réflexions suivies par un ou deux exemples, - car il n'est pas possible d'établir pareille assertion dans l'espace d'un article ${ }^{2}-$, qu'une telle désuétude n'est qu'apparence et que sur bien des points l'épistémologie française d'avant 1950, peut-être parce que mieux équilibrée, reste en avance sur l'épistémologie américaine d'aujourd'hui, même en ses plus récents développements, et que celle-ci par conséquent aurait encore intérêt à étudier son aînée avec attention. Nos réflexions porteront sur la question du but et de la méthode en philosophie des sciences. Nos exemples consisteront à comparer quelques thèses récemment proposées, non sans éclat ni fracas, par Kuhn et Feyerabend, avec quelques idées voisines que l'on rencontrait, quelque trente ans auparavant, chez Gaston Bachelard.

\section{PHILOSOPHIE DES SCIENCES ET PHILOSOPHIE}

La philosophie des sciences est philosophie. Nous entendons par là tout d'abord qu'elle n'est pas une science, au sens où ce terme s'est trouvé défini dans le monde occidental

D'autre part, les signes d'ouverture à d'autres traditions se sont multipliés, ces dernières années, dans la pensée américaine. Mais nous n'avons voulu souligner, dans notre texte, que les dangers philosophiques que comporte une prédomminance linguistique.

2. Pour une argumentation plus détaillée, nous renvoyons à notre travail sur la Pbilosophie française dans la première moitié du XXe siècle qui doit bientôt paraitre chez Privat, à Toulouse, dans un ouvrage collectif dirigé par Pierre Aubenque. 
depuis les XVIIe et XVIIle siècles, c'est-à-dire une discipline qui étudie un secteur déterminé du réel ou du moins, pour inclure dans notre définition (sans avoir à platoniser) les mathématiques, un type d'objet déterminé, selon des critères reconnus de démonstration et de vérification. À première vue, il semblerait que la philosophie des sciences puisse être comprise dans la dénotation d'une définition de ce genre. N'a-t-elle pas un objet déterminé, précisément les sciences? En outre, puisqu'elle cherche en premier lieu à savoir ce que sont les sciences, par conséquent à connaître leurs procédés, leur contenu et leurs articulations avant d'apprécier leur valeur épistémologique et leur fonction humaine, puisque d'autre part les sciences ont une existence de fait, n'est-il pas naturel de croire que la méthode inductive, telle qu'on l'utilise dans les sciences physiques, les sciences humaines ou en histoire, convient parfaitement à cet objet comme à ce but, sans demander rien de plus que cette sorte d'aménagement auquel nous a habitués depuis longtemps le passage d'une science à une autre? Car, on le sait, on ne pratique pas l'induction de la même manière en physique, en biologie et en histoire. Mais, pour peu qu'on réfléchisse davantage sur le but et sur l'objet de la philosophie des sciences, on voit les questions de valeur se mêler inextricablement aux questions de fait. Est-ce sur la science existante, est-ce sur la science idéale que doit porter principalement la réflexion du philosophe? Mais peut-on vraiment les séparer l'une de l'autre, quand on observe tous les jours que l'esprit du savant au travail les unit constamment, et quand on remarque au demeurant qu'il n'y a pas de pratique humaine où n' intervienne un idéal ? Supposons toutefois qu'on parvienne à délimiter, grâce aux œuvres consignées dans les livres, les laboratoires et les musées, le domaine de la science existante, c'est-à-dire la seule qui puisse relever d'une étude empirique. Non seulement nous avons affaire, jusqu'à un certain degré, aux problèmes d'interprétation qui rendent l'histoire si délicate et si problématique, mais nous faisons face à un problème nouveau, sans commun dénominateur avec ceux qu'on rencontre dans les sciences humaines ou en histoire : les questions que le philosophe des sciences pose aux œuvres scientifiques ne sont pas les mêmes en général (malgré l'apparence 
d'importantes exceptions) ${ }^{3}$ que celles que ces œuvres s'efforcent de résoudre ; entre les unes et les autres il y a discontinuité, et même discontinuité plus radicale, en un certain sens, que celle qu'on a coutume d'apercevoir entre la physique et la métaphysique.

Sans doute, quand l'historien examine par exemple les écrits ou les documents signés par Napoléon à Moscou, après l'entrée que celui-ci avait pu croire triomphale dans la Byzance du Nord, il se pose certes des questions fort différentes de celles qui assaillaient alors l'Empereur. Il est responsable d'interprétation, non d'une armée. Il demeure cependant sur le plan des préoccupations et des actions de son héros. Entre les pensées et les actions de l'Empereur telles qu'elles apparaissent à travers ses écrits et l'évocation, le récit ou le commentaire explicatif qu'en fait l'historien, il n'y a pas vraiment discontinuité épistémologique parce que, s'il y a changement dans le mode d'être de l'objet considéré ( $\mathrm{Na}$ poléon et ses adversaires russes sont devenus un passé), l'objet même n'a pas changé. De perception il est devenu souvenir, mais le souvenir a pour matière la matière même de la perception, ce qui fait que l'historien de Napoléon s'occupe au fond et doit s'occuper, bien que sur le mode du récit autant que possible intelligent, des mêmes choses que Napoléon. Or telle n'est pas du tout la relation entre le savant et le philosophe ou plus exactement, - car philosophe et savant, dans l'exercice de leur discipline respective, s'intéressent parfois aux mêmes objets, - entre science et philosophie des sciences. De l'une à l'autre il y a discontinuité épistémologique parce que non seulement l'objet a changé, mais encore, pour parler comme Russell ou comme Pascal, il a changé de type, il a changé d'ordre. La science s'intéresse au réel, la philosophie des sciences à la pensée du réel ; l'une au discours direct, l'autre au sujet ou au symbolisme qui rend tout discours indirect; il est clair qu'on n'a pas affaire au même objet.

3. Les exceptions auxquelles nous songeons ici sont évidemment les questions de logique et de méthodologie. Nous disons qu'elles sont apparentes parce que ces questions ne sont pas envisagées pour elles-mêmes dans le corps des traités scientifiques mais s'y trouvent presque toujours confinées, quand d'aventure elles y sont abordées, dans quelques appendices externes comme les préfaces ou les scholies. 
Cette différence d'ordre, l'épistémologie américaine des trois dernières décades ne l'a certes pas ignorée. Peut-être même l'a-t-elle parfois trop accentuée. Formée pour une large part à l'école du Cercle de Vienne et du premier Wittgenstein, assez fréquemment dans un esprit de stricte obédience mis au compte de la discipline scientifique, elle a en général accepté de ses maîtres une distinction radicale entre la science, - à laquelle on abandonne le tout de la connaissance, entendons tout ce qui peut devenir objet de connaissance, - et la philosophie définie comme pure activité de réflexion, essentiellement d'ailleurs sur la connaissance. ${ }^{4}$ On pourrait avancer, en termes kantiens, que selon une vue de ce genre la science doit prendre pour elle toute la matière de la connaissance, tandis que la philosophie n'en considère que la forme. Mais en vérité ce serait trop dire. Car le terme kantien de "forme" déborde singulièrement le domaine de la logique. Pour les théoriciens du Cercle de Vienne et le premier Wittgenstein au contraire, la pensée, du moins la pensée spéculative, se réduit essentiellement, comme on le sait, à une logique chargée de coordonner en propositions et en discours, en lois et en théories, de façon rationnelle et rigoureuse si elle est bonne, de façon relâchée et parfois paralogique si elle est mauvaise, les données de nos observations ou les éléments de nos constructions. La philosophie des sciences devient ainsi principalement une réflexion sur la logique de la science. Un peu de lecture suffit pour constater, en effet, quelle somme d'examens, quel volume d'encre ont été dépensés en trois décades sur les problèmes de la déduction et de l'induction, de la vérification et de la falsification, de la consistance et de la complétude, des définitions, des axiomes, des postulats, du sens et de la référence, ou sur les raisonnements probabilitaires ; et même quand on aborde des pro-

4. On pourrait nous objecter ce retour vers l'ontologie qu'un Quine a amorcé et qui se continue aujourd'hui dans le sillage d'un Putnam, d'un Lewis ou d'un Kripke. Mais cette ontologie, qu'on voit impliquée dans toute épistémologie, c'est essentiellement aux mathématiques et aux sciences physiques qu'on demande d'en préciser les traits. On ne s'est donc guère éloigné sur ce point des positions fondamentales d'un Russell, d'un Wittgenstein ou d'un Carnap. La science reste la seule autorité compétente en matière d'ontologie. Il va sans dire que cela ne suffit pas à garantir comme "scientifique "l'ontologie que le philosophe, avec ses lunettes particulières, y aperçoit et en dégage. 
blèmes impossibles à confiner dans les perspectives logiques, ainsi sur la nature de la vérité mathématique ou de la vérité en général (Tsarki, Quine, Putnam, Benaceraff), l'originalité des concepts théoriques (Grünbaum, Reichenbach, Carnap) ou le véritable rôle de l'explication (Nagel, Hempel), on tend à les réduire à des questions d'équivalence ou de nonéquivalence entre certains termes. C'est pourquoi d'ailleurs un algorithme symbolique peut être utilisé pour leur solution. Mais quand on s'attache de la sorte à des questions où la technique logique est compétente, c'est-à-dire où la réponse est en principe décidée par le jeu d'un certain nombre de critères, d'axiomes et de règles reconnus, ou bien quand on les pose de façon à ce que cette technique ait juridiction (nous laissons ici de côté la valeur sans doute très variable des solutions apportées), il faut qu'on ait déjà répondu, implicitement sinon explicitement, aux questions fondamentales qui se dressent inéluctablement devant toute épistémologie. Nous visons par là des problèmes de cette nature: quel est le rapport de la pensée au réel, de la perception à l'intellection, de la connaissance commune à la connaissance scientifique, ou encore en quoi consiste, en général ou dans telle science particulière, l'activité de la raison? Il ne semble pas - fautil faire une exception hier pour Quine et aujourd'hui pour Kripke? - que l'épistémologie américaine contemporaine ait produit des œuvres ou même seulement des thèses remarquables sur aucun de ces points. Il est aisé de comprendre pourquoi: l'épistémologie américaine s'est développée à partir de l'épistémologie empiriste et réductionniste du néopositivisme, ou du vieux nominalisme anglo-saxon Wittgensteinisé, acceptés comme cadre principal. Il était admis, en quelque manière sans discussion, par un postulat dont la réception constituait pour ainsi dire le baptême d'entrée dans la communauté philosophique de l'âge scientifique, que ce cadre avait démontré de façon éclatante sa validité et sa supériorité rationnelles. Mais accepter de la sorte, a priori, un tel cadre, c'est peut-être se placer dans de très bonnes conditions pour des exercices, des développements ou des argumentations logiques ; c'est cependant en avoir terminé avec la philosophie.

Les épistémologues français de l'entre-deux-guerres, c'est ainsi que nous nous proposons d'appeler Brunschvicg, 
Meyerson et même Bachelard, ${ }^{5}$ pour ne parler que des plus grands, - ne le cèdent pas, en fait de compétence scientifique, à leurs collègues américains de l'après-guerre qui ne furent pas des savants professionnels. Assurément, pas plus en science qu'en philosophie d'ailleurs, ils n'ont la trempe de Descartes ou de Leibniz; sans doute même ne peut-on leur accorder cette compétence de professionnels qu'avaient eue autrefois un Auguste Comte, un Cournot, un Poincaré ou un Duhem ; en outre, ils sont faibles - mais la plupart des épistémologues contemporains qui s'intéressent à la physique et aux mathématiques ne partagent-ils pas la même faiblesse? - dans le domaine de la biologie et des sciences humaines. Mais en ce qui concerne la chimie, la physique et s'il s'agit de Couturat, de Brunschvicg ou de Cavaillès - les mathématiques, ils connaissent bien l'ensemble de la science classique et suivent la science moderne jusque dans ses derniers développements. Tous ont longuement discuté, par exemple, non seulement de la thermodynamique et de l'électromagnétisme, mais encore de la chimie atomique et de la physique intra-atomique, de la Relativité et des Quanta, dont les théories étaient alors toutes nouvelles. Et chacun propose sur ces sujets, notamment sur la Relativité et les Quanta, quelques vues, sinon quelque interprétation originales. Mais le caractère le plus remarquable de ces diverses épistémologies, c'est qu'elles demeurent pleinement philosophiques.

Prenons une à une, pour nous limiter à celles-là, les trois questions majeures par lesquelles nous avons défini cidessus les buts de l'épistémologie philosophique, à savoir le rapport de la pensée et du réel, le rapport de la science à la connaissance commune, la nature et le sens de l'activité rationnelle. Lisons successivement Brunschvicg, Meyerson et Bachelard avec cette grille (nos conclusions seraient les mêmes si nous ajoutions à nos lectures Édouard le Roy, Lalande, Milhaud, Poirier ou Maritain) : nous obtiendrons des réponses longuement raisonnées, et elles aussi originales, à chacune de ces questions. Ainsi Meyerson établit une conti-

5. Nous mettons Bachelard un peu à part, parce que son cuvre en esthétique n'est guère moins importante, on le sait, que son œuvre en épistémologie. 
nuité entre la connaissance commune et la science, alors que Brunschvicg et Bachelard, de façon différente d'ailleurs, les opposent. Pour Brunschvicg, l'essentiel du réel est ce que l'intelligence parvient à en comprendre mathématiquement, tandis que pour Meyerson, si nous nous permettons de forcer un peu les traits de sa doctrine, ce qui refuse de s'intégrer dans la pensée mąthématique et dans notre logique, rapprochées l'une de l'autre alors que Brunschvicg les opposait. Aux yeux de Bachelard, au contraire, le maximum du réel se situe du côté de ce que les théories et les techniques $\mathrm{du}$ " $\mathrm{Ra}$ tionalisme appliqué " ou du "Matérialisme rationnel " parviennent à construire en partant du possible. Quant à la raison, elle est analytique et relationnelle pour Brunschvicg, identificatrice et spatialisante pour Meyerson, dialectique et synthétique, mais surtout inductive et technique pour Bachelard. Cette sèche énumération ne prétend pas avoir la valeur d'un résumé. Elle voudrait seulement souligner que nous nous trouvons, avec l'épistémologie française de l'entre-deuxguerres, en plein débat philosophique et suggérer que, si on a le courage de longues lectures, - car les épistémologues français ont beaucoup écrit et souvent de gros ouvrages, - ce courage devrait être récompensé.

\section{PHILOSOPHIE DES SCIENCES ET HISTOIRE DES SCIENCES}

La philosopbie des sciences ne saurait être séparée de la philosophie: tel est le premier principe que l'analyse de la notion nous a conduit à dégager et que l'on voit à l'œuvre dans le développement de l'épistémologie française. Formulons-en un second que l'épistémologie américaine la plus récente, en particulier avec Toulmin et Kuhn, semble en voie de retrouver et que l'école française, inspirée par Comte même lorsqu'elle s'en détachait, a toujours mis en application: la philosophie des sciences ne saurait être séparée de l'bistoire des sciences.

Pour établir la validité d'un tel principe, suivons ici la marche inverse de celle que nous avons adoptée pour justifier le principe précédent. En d'autres termes, allons du fait au droit. Dans ce second cas en effet, le fait, à savoir l'usage très 
considérable de l'histoire des sciences par l'épistémologie française, est très connu. Au contraire le droit, à savoir le bien-fondé d'un tel usage s'est trouvé très contesté, notamment dans l'école américaine, à ce point que beaucoup tiennent encore l'histoire des sciences et la philosophie des sciences pour deux disciplines dont il faut maintenir la séparation. Et l'originalité d'un Toulmin et d'un Kuhn, en Amérique, vient précisément de ce qu'ils s'efforcent de réunir à nouveau les deux disciplines dans une philosophie générale de la raison et du progrès scientifiques. ${ }^{6}$

Que l'histoire des sciences, aux yeux des épistémologues français, fasse partie intégrante de la philosophie des sciences, il suffit de parcourir les ouvres et d'entrer tant soit peu dans les pensées pour s'en rendre compte. Le livre qui a consacré la réputation de Brunschvicg comme épistémologue s'intitulait : Les Étapes de la philosophie matbématique, celui qui couronne son œuvre: Le Progrès de la conscience; mais L'Expérience bumaine et la causalité physique se développe d'après le même modèle : en vue d'étudier le passage de la notion de causalité, telle que nous la suggère notre expérience psycho-somatique et la conçoit notre imagination, à cette notion nominalement identique mais intellectuellement toute différente que précise l'intelligence mathématique de l'univers physique, l'auteur retrace les principales conceptions de la causalité depuis Aristote jusqu'à Einstein et Bohr. Sans doute, parce qu'il se propose d'écrire une histoire normative à travers l'histoire réelle, le philosophe prend-il beaucoup de liberté avec cette dernière et, malgré l'abondance des citations, peut-on contester beaucoup de ses interprétations.

6. On trouve un écho de ces débats, par exemple, dans le vol. Ixxi no. 13, July 18, 1874, du Journal of Philosophy à propos des Minnesota Studies in the Pbilosophy of Science, et également dans plusieurs numéros de la revue Science : 14 décembre 1973, vol. 182, no 4117, A Populational Approach to Scientific Change, 1974 passim, ou encore dans le numéro du 25 avril 1975 de la même revue (vol. 188, no 4186): On the Role of Themata in Scientific Thought par Gérard Holton, et la réponse de Robert K. Merton ; cf. aussi sur ce point Th. Kuhn, The Structure of Scientific Revolutions, chap. I "A role for history"). Précisons ici notre pensée : nous ne nions pas que l'histoire des sciences puisse ou même doive constituer une discipline autonome par rapport à la philosophie des sciences; ce que nous nions, c'est que la philosophie des sciences puisse et doive se développer sans s'appuyer constamment sur l'histoire des sciences. 
Quelque appréciation qu'on adopte, il demeure néanmoins hors de doute que la référence à l'histoire constitue, dans l'ouvrage, non seulement une grosse part mais une pièce essentielle. On comprend sans peine, en vérité, qu'il en soit ainsi, puisque pour Brunschvicg la pensée, donc la science, c'est principalement le passage du fait à l'idée, de l'existence à l'essence, de la réalité à l'intelligence (ou, en morale, de l'utilité égocentrique à un idéal universel de perfection) et que ce passage, - qui seul permet de saisir ce qu'accomplit, donc ce qu'est la science par rapport à l'esprit, — s'effectue nécessairement dans une histoire. Meyerson cependant ne partage pas le même optimisme sur le progrès de l'homme et les capacités de la raison. Bien qu'il voit dans la science le métal le plus robuste et le plus précieux qu'ait produit la pensée, il a trop rivé la raison à une tâche d'identification et de réduction à l'espace (ce quasi néant !), ou encore à une "élimination du temps " et de la qualité, pour ne pas apercevoir ce qu'il y a de négatif dans un tel travail; et il ne s'est pas fait faute de le souligner. Mais pour lui également, le travail négatif et passif de la raison (positif parce que l'accroissement en précision quantitative et l'ordination du divers grâce à l'identique représentent un gain incontestable) se poursuit à travers une histoire, sans laquelle d'ailleurs un tel travail n'aurait ni être ni raison d'être. Le principe d'identité, par exemple, ne signifie rien en dehors de l'effort d'identification qui le met en ouvre et par lequel la raison rationalise progressivement l'expérience. Ce qui fait qu'Identité et Réalité offre sans doute la meilleure histoire des principes conservatifs, qu'il s'agisse de la masse, du mouvement, de l'énergie, de la chaleur, de la matière elle-même. Et L'Explication dans les sciences, - comme plus tard l'ouvrage au titre si évocateur pour notre propos Le Cheminement de la pensée, - analyse quelques-uns des principaux modèles d'explication, mécaniques, qualitatifs, dialectiques, successivement utilisés par les sciences ou proposés par les philosophies. Quant à Bachelard, l'homme des coupures et des oppositions épistémologiques, il semble au premier abord beaucoup moins sensible aux leçons du passé, donc à l'histoire. La science moderne ne constitue-t-elle pas à ses yeux un nouvel âge, en quelque sorte aussi révolutionnaire par rapport 
à l'âge classique des Galilée, Newton ou Lavoisier que l'était celui-ci par rapport à la Grèce ou au Moyen-Âge? Car, si l'on en croit Le Nouvel esprit scientifique et $L a$ Philosophie $d u$ non, le rationalisme relativiste et le "surrationalisme" quantique ne diffèrent guère moins du rationalisme classique et de l'empirisme positiviste que ceux-ci ne diffèrent de la connaissance commune. Cependant comment établir et évaluer sans l'histoire le fameux "profil épistémologique " grâce auquel se manifestent les coupures et s'impose ce " pluralisme philosophique" nécessaire à la compréhension, comme il l'a été à la genèse des notions? De fait Bachelard, qui revendiquait pour unique compétence celle de la lecture', n'a pas pratiqué l'histoire d'une façon moins systématique que ses prédécesseurs. Il l'utilise, comme en font foi en particulier Les Intuitions atomistiques et La Formation de l'esprit scientifique, pour sa psychanalyse, entendez aussi sa "catharsis " du savoir. Mais en outre, elle lui est indispensable, comme il l'a dit avec beaucoup de force dans le chapitre I de L'Activité rationaliste de la physique contemporaine, - chapitre dont le titre est significatif : "Les récurrences historiques. Épistémologie et histoire des sciences. La dialectique onde-corpuscule dans son développement historique ", pour désigner et enchaîner les valeurs transhistoriques mêmes, c'est-à-dire les valeurs rationnelles ; celles que la démonstration et l'organisation scientifique de la pensée, sans pour cela les soustraire aux rectifications de la vérification et de la rationalisation en progrès, a rendues permanentes. ${ }^{8}$

En retraçant un fait qui, à l'instar de celui-ci, est un fait philosophique, nous en avons virtuellement, nous semble-t-il, comme établi le droit, dont il nous suffira maintenant de rendre explicites et de condenser les raisons. Personne, en science, n'aurait l'idée de disjoindre les formules abstraites, principes, théorèmes ou lois, des phénomènes concrets dont ils sont l'expression intellectuelle. La relation de la force à la

7. Cf. la prière du "lecteur dévorant" dans la Poétique de la rêverie, pp. 22-23.

8. Cf. aussi cette idée, qu'on pourrait opposer aux vues de Kuhn, développée dans $L e$ Matérialisme rationnel, p. 86 : "Tout historien des sciences est nécessairement un historiographe de la vérité. Les événements de la science s'enchaînent dans une vérité sans cesse accrue (... .). La science grandit. Et c'est cette croissance que l'historien des sciences doit montrer." 
masse et à l'accélération (loi de Newton) ou celle de la différence de potentiel à la résistance et à l'intensité (loi d'Ohm) ne seraient pas seulement, si on essayait de les concevoir en dehors d'une référence à des phénomènes mécaniques ou électriques soit actuels soit réalisables, des relations entre des concepts vides, au sens kantien de ce dernier mot; comme il s'agit de concepts empruntés à la matière de la connaissance et non à sa forme, ces concepts deviendraient autant de termes en quelque sorte privés de signification. Il en est ainsi de l'appareil conceptuel et même logique de la science, dans la mesure immense où celle-ci ne se réduit pas à la logique pure : variables et prédicats, propositions, lois et théories, déduction et induction, vérification, falsification, explication, pour nous en tenir à ces éléments de la science qui appartiennent à l'appareil de la logique, n'ont pas de vraie signification scientifique en dehors de leur usage dans les sciences. Or, ces sciences ont été constituées dans le passé et continuent de nos jours à se développer par un effort indivisiblement historique et collectif. Négliger cet effort, quand on prétend faire la philosophie de la science, c'est se condamner à troquer la science réelle, et même la science idéale telle qu'elle est effectivement pensée par l'humanité, contre un squelette abstrait qui se réduit d'ailleurs au legs desséché d'une réflexion antérieure et dont la vie comme la clarté sont toutes de convention. Mais encore une fois, la science (et ne peut-on dire la même chose au fond de la philosophie et de l'art?) ne se laisse pas plus définir par convention que par imagination personnelle. Qui veut la connaître ou en juger doit donc prendre la matière de réflexion où elle se trouve, c'est-à-dire non pas dans quelque schéma logique que les philosophes astiquent sans fin tandis que les savants les ignorent, mais chez les savants eux-mêmes ou, plus empiriquement encore, dans les ouvres par lesquelles la science effective se communique et se conserve.

Objectera-t-on alors qu'on nous accorde ce point, mais que la science intéressante pour l'épistémologue est la science vivante, non la science du passé, qui convient aux recherches de l'historien plutôt qu'aux objectifs du philosophe? Mais d'abord il est très difficile, pour ne pas dire arbitraire, de 
dissocier la science vivante de la science passée. Plus que jamais, en raison même de l'immense acquis dont se compose la science contemporaine, la définition aristotélicienne de la science comme habitus s'avère pertinente. Qu'est-ce qui introduit le futur savant à la science en train de se faire, celle des observatoires et des laboratoires, sinon l'assimilation de nombreux et volumineux manuels, où on a condensé et systématisé la science acquise? Acquise quand? Dans le passé. Ainsi apprendre une science, c'est assimiler dans ses résultats sinon dans son devenir l'histoire de cette science. Admettons que le savant, par intérêt et nécessité de son état, s'attache moins, dans la généralité des cas, au devenir qu'aux résultats. Mais le philosophe ne partage tout à fait ni les mêmes intentions ni la même situation. De son côté, dans la majorité des cas il n'est pas et ne peut être un savant : l'extrême spécialisation et surtout l'extrême complication dans la spécialisation qui caractérisent les sciences modernes l'en empêchent, du moins en règle générale. Par conséquent, il ne bénéficie pas de cette espèce de connaissance directe - la fameuse " acquaintance " de Russell - que procurent la familiarité et la pratique. Raison de plus pour lui de s'instruire sur la science auprès des savants; or, on peut affirmer sans paradoxe que le savant du passé se laisse plus facilement observer que le savant contemporain. En outre, le rapport de la fonction scientifique à l'esprit intéresse le philosophe encore plus que le rapport de la connaissance ne disons pas au réel dans son ensemble, lequel est un tout premier objet de philosophie, mais à ce secteur particulier du réel qui forme l'objet propre de chaque discipline spécialisée. Or, le rapport de la fonction scientifique à l'esprit s'est dessiné à travers une longue suite d'histoires, individuelles et collectives. Sans référence précise à cet ensemble historique, on peut bien l'apercevoir. Enfin, en raison du rôle synoptique qui est dévolu depuis toujours à la philosophie, le philosophe considère en priorité les notions cardinales, les principes, les théories générales et les découvertes majeures, qui de par eux-mêmes confèrent déjà à la science une insigne valeur philosophique. Or dans le domaine des découvertes, des théories et des notions fondamentales, il y a une sorte de privilège de l'intention. Le génie de Descartes éclaire d'un jour incomparable la géomé- 
trie analytique, celui de Newton et de Leibniz la mécanique rationnelle et le calcul infinitésimal, celui d'Einstein la géométrie et la mécanique relativistes, celui de Darwin l'évolutionnisme ou le génie collectif du XVIIIe siècle, etc. ${ }^{9}$ Pourquoi le philosophe n'irait-il pas chercher dans l'histoire des sciences la philosophie que celle-ci contient et découvre...., mais seulement à l'œil qui s'équipe pour voir?

\section{SI ON RAPPROCHAIT LES DEUX TRADITIONS}

La méditation générale a mordu largement sur la place que nous pensions réserver aux exemples. Il est temps d'évoquer brièvement ceux que nous avons choisis. Le lecteur en poursuivra, s'il lui plaît, l'analyse et en cherchera d'autres.

Nos exemples visent à illustrer notre thèse générale : les épistémologues américains gagneraient, et auraient déjà gagné dans un récent passé, à connaître davantage les travaux de leurs homologues ou prédécesseurs français. Nous ne tenons pas moins à la réciproque: mais l'abondance de la production américaine, en ce domaine également, l'a rendue si obvie qu'il serait ridicule d'y insister. On ne montre pas au pauvre l'intérêt qu'il a à recevoir du riche; en revanche il peut n'être pas inutile d'attirer l'attention du riche s'il est réputé généreux, sur les valeurs du pauvre. Le riche évidem. ment généreux, puisqu'il s'agit de philosophes, c'est le vaste ensemble des épistémologues américains, tandis que l'épistémologie française est devenue plutôt pauvre. Mais ce pauvre, auquel un brillant avenir n'est d'ailleurs pas interdit, a des trésors dans son héritage. Et parmi les épistémologues américains que leurs principes, leur orientation et leur formation rapprochaient davantage de la tradition française, et qui en vérité ont su à l'occasion marquer leur reconnaissance envers un Koyré, un Meyerson ou un Duhem, plusieurs d'entre eux

9. De façon analogue, mais à certains égards inverse, l'histoire des sciences pourrait permettre aussi de mettre en relief l'aspect négatif des découvertes empiriques et surtout des mutations théoriques : ainsi, par exemple, ce qu'on a perdu en abandonnant l'aristotélisme pour le cartésianisme, les ondes pour les corpuscules ou viceversa, ou quand on a découvert le rôle des microbes dans les maladies, ou peut-être de nos jours le rôle de l'A.D.N. dans la formation de l'organisme. L'histoire des sciences peut inviter à une philosophie plus équilibrée, nous dirions même (au risque d'une redondance apparente) plus sage de la science. 
eussent peut-être avancé plus vite et plus loin sur leur propre voie s'ils avaient mieux connu les auteurs que nous avons évoqués ci-dessus. Nous allons essayer de le montrer sur un ou deux exemples précis, en confrontant quelques idées de $\mathrm{Kuhn}$ et de Feyerabend avec quelques idées analogues de Bachelard exprimées quelque trente ans auparavant.

Assurément le lecteur français de The Structure of Scientific Revolutions découvrira dans ce livre des idées qu'il n'a jamais vues énoncées, du moins sous cette forme, chez les auteurs qui lui sont familiers, et en particulier chez Gaston Bachelard. Plus précisément, par exemple, il n'aura pas rencontré chez le philosophe français la distinction si nette, à vrai dire poussée jusqu'à l'opposition, entre la science ordinaire - " normal science " - et la science en révolution "scientific revolutions" - (à ce point qu'il est difficile de traduire les termes), distinction proclamée par le brillant épistémologue américain. Pour l'auteur de La Connaissance approchée, aux yeux de qui la science du laboratoire et de la théorie doit se maintenir en un perpétuel mouvement de rectification et d'approximations moins accrues qu'affinées et mieux comprises, ${ }^{10}$ ce que Kuhn appelle " normal science " ne saurait se réduire à un travail de "puzzle-solving ", car il s'y poursuit, au contraire, par cet approfondissement et par le remaniement des concepts qu'entraîne presque toujours l'accroissement des connaissances en extension, en complexité et en précision, la création des cadres de la pensée. En d'autres termes, l'invention notionnelle et théorique sans laquelle il n'y aurait pas de progrès scientifique n'est pas du tout, pour Bachelard, l'apanage des révolutions très apparentes de la pensée : elle s'affirme aussi continuellement jaillissante (ne disons pas continue: Bachelard, on le sait, n'aimait pas le continu) que la multiplication du progrès technique, sans lequel il n'y aurait pas non plus progrès scientifique. Bref le philosophe qui a grandi entre la Champagne et la Bourgogne, en un pays où les villes se mêlaient hier encore aux jardins et aux vergers, a cru du génie d'invention théorique ce qu'il a

10. Cf. à ce sujet le chap. I de La Valeur inductive de la relativité et le chap. XI ("Les obstacles de la connaissance quantitative ") de La Formation de l'esprit scienti. fique. 
maintes fois répété du génie poétique : il est plus répandu qu'une histoire sommaire des " grandes œuvres " ne le laisse imaginer ; il brille, fleurit et fructifie même en des esprits où sa sève n'a pas atteint le degré de condensation et de puissance qu'elle manifeste en d'autres. C'est ainsi que, si l'on s'en rapporte à l'un des tout premiers livres de notre auteur, Étude sur l'évolution d'un problème de physique, la propagation thermique dans les solides, ${ }^{11}$ ce chapitre important de la Thermodynamique a franchi sans révolution aucune, des premiers calculs de Biot aux équations de Fourier, de celles-ci aux constructions de Poisson et aux recherches plus spéciales de Duhamel, enfin de la "synthèse mathématique " de Lamé aux théories cinétiques de Boussinesq, plusieurs étapes épistémologiques dont chảcune en son genre constitue une véritable innovation théorique, et parfois technique, par rapport à la précédente. De ce point de vue, le philosophe français n'anticipe point, mais contredit plutôt les idées du philosophe américain. Pareillement, la notion de "paradigme ", centrale chez ce dernier, ne bénéficie, du moins sous ce vocable, que de quelques allusions chez le premier où l'on ne rencontre que des analogues plus ou moins proches, ainsi les "nœuds", ou les "interconnexions de concepts ", étudiés en particulier dans les derniers ouvrages. ${ }^{12}$

Mais un trait essentiel de l'épistémologie de Bachelard anticipe de plus près les thèses de Kuhn sur les révolutions scientifiques, c'est-à-dire sur les changements de paradigmes à l'intérieur d'une science ou d'un ensemble de sciences. Il s'agit, on l'a deviné, du rapport dialectique que l'auteur de La Pbilosopbie du non établit non seulement entre les époques dēla pensée scientifique, c'est-à-dire entre les théories les plus générales et les philosophies qui les inspirent, mais encore, dans le cours d'une même époque ou dans l'optique d'une même théorie, entre les phases successives de son développement. ${ }^{13}$ La dialectique des philosophies, que résume

11. Ouvrage que Bachelard avait présenté comme thèse complémentaire de doctorat, en 1927, L'Essai sur la connaissance approchée étant la thèse principale.

12. Plus précisément dans Le Rationalisme appliqué (p. 144, " les corps de concepts"), et surtout $L$ 'Activité rationaliste de la pbysique contemporaine, passim.

13. À ce sujet notre collègue de Montréal monsieur Nadeau, nous a fait, lors de notre conférence de Moncton, une objection qui ne manque ni de pertinence ni de force : 
le célèbre profil épistémologique de La Pbilosophie du non et dont Le Nouvel esprit scientifique avait déjà donné un aperçu dans un style plutôt fracassant, est familière aux lecteurs philosophes. Ceux-ci, comme pour éviter de tomber parmi ses victimes, ont le plus souvent pris le parti de l'admirer, car un des buts avoués de cette dialectique était de reléguer la foule des philosophes parmi les formes inférieures de la pensée (aux premiers "âges de l'intelligence" pour employer les catégories de Brunschvicg), en laissant entendre qu'ils s'attardent encore au réalisme, au substantialisme ou à l'intuitivisme du sens commun, tandis que la science a maintenant franchi les étapes de l'empirisme, du rationalisme classique et du rationalisme relativiste pour en arriver au surrationalisme discursif qui dialectise dans des totalités de plus en plus unifiées mais de plus en plus complexes les concepts de la raison et les éléments du réel. Cette dialectique philosophique du profil épistémologique ne nous paraît, en vérité, ni la plus originale ni la plus remarquable. On en trouve l'analogue un peu partout d'Auguste Comte à Brunschvicg, avec une justification théorique chez ces derniers auteurs qui demeure presque absente chez Bachelard; et celui-ci n'a guère eu d'autre mérite que de baptiser la dernière étape d'un mot destiné à faire effet à une époque où le surréalisme et le surmoi étaient en vogue, puis de jouer de son "profil " avec une grande habileté publicaine, publicitaire ou polémique.

il n'y a pas de dialectique chez Kuhn, comme le soulignent d'ailleurs les remarques, qu'à certains égards un sceptique pourrait faire siennes, du dernier chapitre de The Structure of Scientific Revolutions: "Progress through revolutions". Il nous semble que nous avons nous-même répondu à cette objection, en y faisant droit dans les dernières pages de notre article : la dialectique, ou plus exactement l'effort de synthèse qui s'y déploie, permet à Bachelard de sauvegarder l'unité du vrai, donc la réalité du progrès scientifique. Et c'est là, à nos yeux, une supériorité de l'épistémologie du philosophe français. Ceci dit, la dialectique n'a pas du tout chez Bachelard une fonction d'anticipation, et elle ne se développe pas, comme chez Hegel ou même chez Hamelin, selon une logique de la nécessité. Elle se manifeste essentiellement, d'abord, par une coupure ou une rupture, par un " non " qui ne détermine nullement le "oui "subséquent. La synthèse se fait après coup, lorsque la "valeur inductive " des nouvelles théories, qui permettent de rejoindre les anciennes à partir d'une compréhension et d'une extension supérieures, se trouve aperçue. C'est pourquoi la partie initiale de la phénoménologie dialectique de Bachelard cö̈ncide assez bien, compte tenu des réserves que nous avons faites à propos de la "science normale", avec la phénoménologie mutationniste de Thomas Kuhn. 
Par suite, ce n'est pas de ce côté que nous situerions l'anticipation la plus originale des idées de Kuhn. Nous la verrions davantage du côté de ces dialectiques plus subtiles et plus difficiles, mais aussi plus véritables, selon lesquelles l'épistémologue français décrit les révisions, les étapes, enfin les crises successives qui ont scandé jusqu'à nous le progrès scientifique. ${ }^{14}$ Car le "non " a finalement autant de formes que les questions qui le provoquent et les concepts auxquels il s'oppose. Il y a un pluralisme du "non " comme il y a un pluralisme philosophique des notions. Et ici, nous semble-t-il, l'é. pistémologue français prend décidément l'avantage, au plan de la phénoménologie même, sur l'épistémologue américain. Car depuis La Connaissance approchée, où déjà il montrait comment l'approximation, parce qu'elle doit être pensée, entraîne sans cesse des rectifications qualitatives (en provoquant soit la considération, soit l'élimination, soit l'épuration du détail, tantôt la simplification et tantôt la complication), jusqu'au Matérialisme rationnel, qui souligne l'unité toute moderne entre une raison réaliste et une matière rationalisée, ce n'est pas un ou deux exemples, ce sont des dizaines d'exemples que le philosophe soumet à l'analyse. Que cette dernière s'avère parfois rapide, parfois même plus suggestive que démonstrative, nous n'en disconviendrons pas. Il y aurait donc lieu de se demander si les fameuses "coupures épistémologiques" sont toujours aussi vives que le déclare Bachelard, surtout si elles ont toujours la signification qu'il leur attribue. Un tel examen déborderait de beaucoup le cadre et le propos de notre communication. Nous serions heureux si, par les remarques précédentes, nous avions encouragé quelqu'un à l'entreprendre. Pour le moment, il nous suffira d'avoir indiqué comment l'idée centrale de Kuhn avait été anticipée, de façon supérieure à bien des égards, par le dernier des grands épistémologues français. Écoutons-le parler. Quelques lignes tirées de son plus célèbre ouvrage's (qui est loin d'être

14. Les textes les plus remarquables à cet égard nous paraissent, outre les thèses de doctorat déjà citées, Les Intuitions Atomistiques, Le Pluralisme cobérent de la Chimie moderne et L'Activité rationaliste de la Physique contemporaine, où Bachelard retrace en particulier la dialectique de la notion d'atome, celle d'espèce chimique et celle de corpuscule.

15. Le Nouvel esprit scientifique, p. 134. 
le meilleur à nos yeux) diront mieux quelle conception précise il se formait des révolutions scientifiques: "Quelle que soit la durée du repos dans le réalisme, ce qui doit frapper, c'est que toutes les révolutions fructueuses de la pensée scientifique sont des crises qui obligent à un reclassement profond du réalisme. De plus, ce n'est jamais la pensée réaliste "- entendons la seule observation empirique - "qui provoque ses propres crises. L'impulsion révolutionnaire vient d'ailleurs : elle prend naissance dans le règne de l'abstrait. C'est dans le domaine mathématique que sont les sources de la pensée expérimentale contemporaine ".

Ce que nous venons de faire pour l'idée de révolution scientifique, nous pourrions le répéter aisément, avec plus d'exactitude encore, pour une autre idée cardinale chez Kuhn, celle qui souligne non seulement la rupture entre la science et le savoir préscientifique, mais encore le caractère nécessairement collectif et social de la "science normale ", œuvre d'une communauté scientifique hautement spécialisée. Dans les trois grands ouvrages d'épistémologie qu'il a publiés après la deuxième guerre mondiale, de 1948 à 1953 précisément, Bachelard, qui devient avec Teilhard de Chardin le chantre de "la cité scientifique " et le défenseur de la spécialisation, a insisté avec beaucoup de force et de détail sur tous les aspects de cette même idée. En cela consiste d'ailleurs la principale originalité de ces livres nouveaux par rapport aux précédents, puisqu'ils se proposent tous d'illustrer, en analysant les récents développements de la physique et de la chimie intra-atomiques, les nouvelles formules lancées par le premier d'entre eux (Le Rationalisme appliqué) : à savoir le "Cogitamus", que la science moderne aurait substitué au Cogito des philosophes, et le "Corationalisme " qu'est devenu le surrationalisme grâce à " l'Union des Travailleurs de la preuve". "Cette socialisation intense, clairement cohérente, sûre de ses bases, ardente dans ses différenciations, voilà encore un fait, un fait d'une singulière actualité (. . . .). Le départ culturel de la science précise désormais tout départ naturel. Être un chimiste, c'est se mettre en situation culturelle, en prenant place, en prenant rang, dans une cité scientifique nettement déterminée par la modernité de la recherche. 
Tout individualisme serait un anachronisme. ${ }^{16}$ On trouverait sans peine des dizaines de textes dans la même veine, où les idées de Kuhn sur la science comme discipline sociale - à part l'idée de "puzzle-solving " que Bachelard n'accepterait pas sans réserves expresses - sont déjà, quinze ou vingt ans auparavant, nettement formulées. La grosses différence, analogue à celle qu'on remarque au niveau de la dialectique du "non ", où les coupures successives n'empêchent pas l'organisation irréversible et croissante des valeurs rationnelles, c'est-à-dire des valeurs permanentes, est que pour le philosophe français le caractère social de la science moderne fournit un argument non pas à un certain pragmatisme des théories, mais au rationalisme de la vérité. Quand "on passe de la vérité vérifiée à la preuve prouvée ${ }^{17}$, apanage de la communauté scientifique, on voit mieux comment la systématisation inscrite dans une auvre collective élimine l'aberration individuelle, donc accroît le caractère "réalistique", voire "nouménal " de la pensée scientifique ; et on aperçoit plus clairement aussi que "la raison a des thèmes de fidélité ", " qu'en découvrant le vrai, l'homme de science barre un irrationnel " et que "l'histoire des sciences est l'histoire des défaites de l'irrationalisme ". Cette divergence d'interprétation a le mérite de maintenir ouvert, à propos d'une phénoménologie presque identique et au demeurant peu constable, un débat philosophique dont l'enjeu est considérable.

Nous ne contesterons pas davantage l'originalité de Feyerabend, par rapport à Bachelard, soit sur l'avenir de la science en général, soit sur la physique quantique en particulier. Cette orignalité apparâit même dans l'article manifeste How to be a God Empiricist ${ }^{19}$ que nous prenons pour point de. référence, et qui n'est cependant qu'un résumé de travaux antérieurs, notamment sur l'empirisme et la microphysique. En un mot, Feyerabend est las du dogmatisme empiriste et positiviste, sinon agnostique, qui s'est greffé sur la théorie des Quanta telle qu'elle a été interprétée selon les relations d'incertitude d'Heisenberg et surtout l'épistémologie néo-

16. Le Matérialisme rationnel, p. 2.

17. L'Activité rationaliste de la physique contemporaine, p. 28.

18. Ibid., pp. 26-27.

19. Cf. The Philosophy of Science (Oxford readings in Philosophy), pp. 12-39 
positiviste du Cercle de Vienne. Il voudrait rouvrir la porte à des interprétations comme celles de Bohm, de de Broglie et de Vigier, c'est-à-dire en somme réintroduire dans la théorie quantique certaines formes de déterminisme et l'explication rationnelle. Bachelard, au contraire, n'a jamais senti dans la physique quantique une menace pour le rationalisme, mais bien plutôt une promotion du rationalisme: la regardant à travers l'école de de Broglie plutôt qu'à travers l'école de Copenhague, et n'ayant jamais fait du déterminisme une norme (il y voyait en vérité, quand on le conçoit comme universel, une dangereuse idole, et ne croyait, ainsi qu'il l'a dit très clairement dans L'Activité rationaliste de la physique contem. poraine, qu'aux déterminismes régionaux), il se réjouissait, avec une sorte de jubilation intellectuelle, de la multiplication des entités corpusculaires, de l'éclatement des notions classiques, de l'usage des oppositions dialectiques et plus encore peut-être du discontinu. C'était là, pour lui, autant de preuves qu'avec la Physique des Quanta la raison humaine avait atteint comme un sommet d'où elle apercevrait de mieux en mieux ou continuerait à construire l'architecture rationnelle de l'univers; de là le nom glorieux par lequel il la magnifie, "surrationalisme dialectique et discursif ". L'opposition de sentiment, sinon de jugement, entre les deux philosophes est donc, derechef, très grande. Mais alors les analogies qu'on peut noter entre leurs pensées n’en deviennent que plus remarquables, d'aurant que ces analogies tiennent à la conception que se font les deux auteurs des concepts et des théories scientifiques, c'est-à-dire à leur épistémologie générale.

Afin de rouvrir la porte à un développement plus rationaliste de la physique antique, lequel coïnciderait avec un empirisme de meilleur aloi, celui d'un " good empiricst ", Feyrabend attaque en effet dans son article ${ }^{20}$ les deux postulats épistémologiques que les théoriciens du groupe de Copenhague et les philosophes du Cercle de Vienne ont mis en avant pour maintenir la physique des Quanta dans l'orbe du calcul statistique et probabilitaire sur le plan scientifique, du néopositivisme et en fin de compte du nominalisme sur le plan philosophique. Il s'agit des deux conditions que Feyerabend

20. C'est toujours à How to be a Good Empiricist que nous faisons allusion. 
appelle, en anglais, la " consistency condition " et la " condition of meaning invariance ", termes que nous proposerions de traduire par "compatibilité logique " et "constance sémantique ", à condition de conserver à ce dernier mot le sens que lui donnent ordinairement les grammairiens. Le premier postulat concerne le rapport des théories et il signifie qu'une théorie nouvelle doit s'accorder avec les théories que l'opinion savante, fondée sur la logique et l'expérience, juge bien établies. Le deuxième postulat concerne le sens des termes et il demande que lorsque des termes ont reçu une signification précise dans la science établie (c'est-à-dire en particulier désignent des faits certains ou des notions bien définies), leur signification ne soit pas altérée par les théories qui prétendent en expliquer de façon nouvelle le contenu. Feyerabend juge ces deux conditions "restrictives", car elles imposent à la pensée un joug trop étroit et conviendraient mieux à une théologie opiniâtrement conservatrice et dogmatique qu'à une science animée d'esprit critique et désireuse de progrès. En bon dialecticien qui retourne contre l'adversaire les armes de celui-ci, l'auteur de How to be a Good Empiricist montre alors contre l'empirisme offficiel que ces conditions restrictives n'ont, en fait, jamais été observées par la science et il illustre sa thèse par deux exemples frappants : $1^{\circ}$ l'opposition que la théorie cinétique de la chaleur, présentée comme une "alternative " à la conception positiviste de la thermodynamique, permet d'établir entre le mouvement brownien et la deuxième loi de la thermodynamique, que Feyerabend considère comme "réfutée " par là ; $2^{\circ}$ l'opposition syntaxique et sémantique entre la physique relativiste et la physique newtonienne, qui fait, par exemple, que le concept de masse ne conserve pas la même signification et ne permet pas les mêmes opérations lorsqu'on passe d'une théorie à l'autre. On peut conclure : la science progresse grâce aux alternatives que proposent, en face des faits, les théories opposées; et, pour favoriser le progrès scientifique, il faut favoriser tout d'abord, au lieu de la restreindre, la production d'alternatives théoriques.

Or plus de trente ans auparavant, et très précisément à propos de la Relativité dont il voulait corriger l'interprétation 
meyersonienne, ${ }^{21}$ Bachelard proclamait avec beaucoup d'éclat, et pour ainsi dire dans les mêmes termes, des idées analogues. Ici encore il suffit de le lire pour s'en convaincre. Résumant, dans Le Nouvel esprit scientifique, l'analyse épistémologique qu'il avait développée en 1929 dans $L a$ Valeur inductive de la relativité, il écrit en effet sur ce qu'il appelle (et l'expression a une valeur symbolique) "la mécanique non-newtonienne ": "Il ne s'agit pas de mots qui changent de sens tandis que la syntaxe serait invariable, pas davantage d'une syntaxe, mobile et libre, qui retrouverait les mêmes idées à organiser. Les relations théoriques entre les notions modifient la définition des notions autant qu'une modification dans la définition des notions modifie leurs rela-

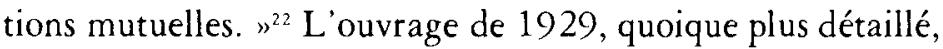
est plus net encore s'il est possible. L'assertion liminaire : "Un des caractères les plus évidents des doctrines relativistes, c'est leur nouveauté " revient par la suite comme un leitmotiv qui scande les étapes de l'analyse. Ainsi p. 25 : «En résumé, examinés du simple point de vue du nombre, - principe si apte pourtant à effacer des différences, les deux systèmes newtonien et einsteinien paraissent sans ressemblance, sans lien, sans parenté inductive. Le système ancien n'est pas naturellement continué par le système moderne. " Et p. 43 : “On ne voit rien dans le système de Newton qui puisse faire prévoir le système d'Einstein (....). Aucune voie d'inférence ne permet d'avancer du premier au second ", d'autant que "si le système d'Einstein ne visait qu'à perfectionner la réussite numérique, ce ne serait peut-être pas la peine de bouleverser les habitudes de notre pensée pour augmenter la précision atteinte dans le système de Newton ". De même on ne passe pas du système d'Einstein à celui de Newton par une simple diminution d'exigences en matière d'approximation, car " en faisant abstraction d'une courbure ", si faible soitelle, "le système (newtonien) méconnaît un caractère essentiel " dans le système relativiste (p. 45). Enfin pp. 182-185: "Le succès de la Relativité est d'abord la conscience nette

21. Le titre même de l'ouvrage de Bachelard, La Valeur inductive de la relativité, forme une antithèse avec celui de Meyerson, publié quatre ans plus tôt en 1925, La Déduction relativiste.

22. Le Nouvel esprit scientifique, chap. I, "La mécanique non-newtonienne, "p. 51. 
d'un échec des théories classiques (. . .). La Relativité ne continue pas les doctrines anciennes, elle les rectifie (...). (Elle) est venue réveiller le savant d'un scepticisme affirmé avec une tranquillité dogmatique ", puisqu'elle est née dans l'ère "des tolérances de la commodité ". Bref, aux yeux de Bachelard, la Relativité générale poursuit le remaniement fondamental et théorique commencé par la Relativité restreinte, car les deux, prises ensemble, altèrent ou plutôt révolutionnent les notions primitives de la mécanique newtonienne, espace, temps, gravitation, force, masse, énergie, de même que les principes d'équivalence qui les lient et la structure d'univers qu'elles décrivent. Il s'agissait donc de tout autre chose que d'un accroissement de l'approximation jusqu'à la huitième décimale, alors qu'on n'atteignait encore que la quatrième en laboratoire, ou d'une récupération des 43 " d'erreur dans la variation annuelle du périhélie de Mercure, laquelle erreur ne constituait pas un grave échec pour la physique newtonienne. Plus exactement, le changement d'ordre dans le degré de l'approximation montrait qu'on avait affaire à un changement qualitatif, voire essentiel, dans le domaine des principes mécaniques et même du cadre épistémologique: on se trouvait en présence d'une véritable alternative théorique de portée épistémologico-ontologique toute nouvelle, et au sujet de laquelle une expérience réorganisée autant qu'affinée était appelée à trancher. N'avons-nous pas ici, dans un développement beaucoup plus circonstancié que notre évocation ne le laisse imaginer, les vues et les desiderata récemment exprimés par Feyerabend?

Mais à propos de cette "épistémologie de l'alternative ", dont Bachelard empruntait d'ailleurs l'idée à l'épistémologue anglais Campbell (heureux temps où les philosophies "insulaire " et " continentale " communiquaient si aisément), notre auteur prend bien soin d'écrire: "Il ne suffit pas de proposer des alternatives, il faut montrer qu'elles se posent nécessairement, par la force de la généralisation, par la vie même de la pensée inductive. Ainsi coordonnées, les alternatives réservent la raison de leur totalisation. Elles passent au rang des faits. Le doute se présente comme le premier signe d'élargissement de la doctrine. L'alternative 
bien équilibrée donne ensuite le plan de la possibilité. Une méthode qui n'était qu'éristique devient heuristique dès qu'on a compris la nécessité profonde de la controverse. ${ }^{23}$ Ajoutons, car tel est bien, nous l'avons vu, la pensée de Bachelard: d'une controverse ordonnée à la croissance de la vérité et de la rationalité. N'y a-t-il pas là, dans cette réserve du bon sens, de quoi prévenir les inquiétudes, légitimes à nos yeux, que pourrait faire naître, par contraste avec l'académisme néo-positiviste, un certain romantisme de l'alternative décelée par certains, non sans raison peut-être, chez Feyerabend? L'équilibre intellectuel et moral dont la tradition française avait fait une vertu retrouverait ainsi une éminente valeur épistémologique.

Nous conclurons en deux mots cette libre promenade à travers quelques thèmes et quelques textes, laquelle se proposait moins l'apologie d'une tradition qu'un plaidoyer en faveur de la communication réciproque des cultures et des recherches philosophiques. Si les réflexions et les exemples précédents ont quelque force probatoire, on peut en retenir en effet une double morale. Il serait utile à la philosophie en général, et à la philosophie des sciences en particulier, de relier plus étroitement et plus constamment les débats du présent avec les grands textes apparentés qui méritent de survivre dans la mémoire humaine; car les philosophies, comme la science, ne seraient rien si elles n'avaient la force de résister au temps qui passe. En second lieu, le français, même pour la philosophie en Amérique, ne doit pas être compté parmi les pièces de musée, mais doit être tenu, en raison de l'actualité de son récent passé philosophique, pour une langue d'avenir. Ou du moins, pour qu'il le soit, il suffit qu'on le veuille.

23. La Valeur inductive de la relativité, chap. V, "Les garanties d'unité de la doctrine, " p. 181. 